\title{
Vibrational dynamics up to the dissociation threshold: A case study of two-dimensional $\mathrm{HOCl}$
}

\author{
Marc Joyeux, Dominique Sugny, and Maurice Lombardi \\ Laboratoire de Spectrométrie Physique, Université Joseph Fourier-Grenoble I, BP 87, \\ F-38402 St Martin d'Hères, France \\ Remy Jost \\ Grenoble High Magnetic Field Laboratory, MPI-FKF and CNRS, BP 166, F-38042 Grenoble, France \\ Reinhard Schinke \\ Max-Planck-Institut für Strömungsforschung, D-37073 Göttingen, Germany
}

Sergei Skokov and Joël Bowman

Department of Chemistry and Cherry L. Emerson Center for Scientific Computation, Emory University, Atlanta, Georgia 30322

(Received 7 July 2000; accepted 7 September 2000)

\begin{abstract}
This work is aimed at extending recent studies dealing with the highly excited vibrational dynamics of HOCl [J. Chem. Phys. 111, 6807 (1999); J. Chem. Phys. 112, 77 (2000)], by taking advantage of the fact that the $\mathrm{OH}$-stretch remains largely decoupled from the two other degrees of freedom up to and above the dissociation threshold. The molecule is thus reduced to a two-dimensional (2D) system by freezing the $\mathrm{OH}$ bond length to its equilibrium value. All of the calculated bound states of the 2D system, as well as the first 40 resonances, can be assigned with a Fermi polyad quantum number. The bifurcation diagram of the principal families of periodic orbits (POs) is extended to higher energies compared to 3D studies. In particular, the birth of "inversion" states (states exploring two equivalent wells connected through the linear $\mathrm{HOCl}$ configuration) is related to a period-doubling bifurcation of the families of bending POs, while "dissociation" states (states for which the energy flows back and forth along the dissociation pathway) are shown to lie on top of three successive families of POs born at saddle-node bifurcations. Based on the derivation of a classical analogue of the quantum Fermi polyad number, the energies of particular quantum states and classical POs are plotted on the same diagram for the 2D ab initio surface and are shown to agree perfectly. In contrast, comparison of classical Poincaré surfaces of section and quantum Husimi distributions suggests that the classical dynamics of $2 \mathrm{D} \mathrm{HOCl}$ is much more chaotic than the quantum dynamics. This observation is discussed in terms of the quantum/classical correspondence, and particularly of the vague tori introduced by Reinhardt. It is nevertheless shown that quantum and classical mechanics agree in predicting a slow intramolecular vibrational energy redistribution (IVR) between the $\mathrm{OCl}$ stretch and the bend degrees of freedom. (C) 2000 American Institute of Physics. [S0021-9606(00)00945-4]
\end{abstract}

\section{INTRODUCTION}

Recent theoretical and computational studies of the dynamics of $\mathrm{LiCN},{ }^{1,2} \mathrm{HCP},{ }^{3-6} \mathrm{HOCl},{ }^{7,8} \mathrm{C}_{2} \mathrm{H}_{2},{ }^{9,10} \mathrm{DCP},{ }^{11}$ and $\mathrm{SO}_{2}{ }^{12}$ have clearly shown the benefit of classical analyses for revealing and understanding the information relative to highly excited vibrational dynamics contained in fitted or $a b$ initio quantum mechanical spectra. In particular, classical bifurcations (that is, in general, the sudden birth of new families of fundamental periodic orbits) were shown to be responsible for the appearance of new families of quantum states, which are precursors of fundamental processes, such as isomerization ${ }^{1-6,9,10}$ and dissociation. ${ }^{7,8}$

In particular, $\mathrm{HOCl}$ has recently been the subject of much interest, both from the experimental ${ }^{13-20}$ and theoretical $^{7,8,21-29}$ points of view. Potential energy surfaces (PESs) with near spectroscopic accuracy, suitable for accurate calculation of high-energy vibrations and dynamics stud- ies, have been developed. ${ }^{8,21,26,27}$ The wave functions of all bound states up to the $\mathrm{HOCl} \rightarrow \mathrm{HO}+\mathrm{Cl}$ dissociation threshold at $D_{0}=19290 \mathrm{~cm}^{-1}$ above the ground state (there are more than 800 of them) were visually inspected, in order to assign the states and to elucidate how the spectrum develops with energy. ${ }^{7,8}$ It was shown that the dominant feature is a $2: 1$ anharmonic resonance between the bending mode and the $\mathrm{OCl}$ stretching motion. This resonance is responsible for a saddle-node bifurcation, which occurs at about $13000 \mathrm{~cm}^{-1}$ above the ground state for states with no excitation in the $\mathrm{OH}$ stretch. The family of stable periodic orbits (POs) born at this bifurcation closely follows the dissociation pathway up to very high energies, while the family of POs, which follows this pathway at lower energies, progressively curves and avoids dissociation. ${ }^{7,8}$ Consequently, the "new" quantum states which appear above $13000 \mathrm{~cm}^{-1}$ and which lie on top of the new family of POs, were called "dissociation" states in Ref. 8. It was furthermore shown that all of the 
features observed in the ab initio spectrum up to $98 \%$ of the dissociation energy, including the bifurcation and the birth of the dissociation states, could be reproduced with excellent accuracy by a fitted Fermi resonance Hamiltonian, that is, a Hamiltonian which takes into account only the 2:1 resonance between the bending and the $\mathrm{OCl}$ degrees of freedom. ${ }^{7}$ This Fermi resonance Hamiltonian was used to rationalize the shape of the quantum wave functions in terms of the position of the state in the quantum Fermi polyads. ${ }^{7}$

The goal of the present work is to take advantage of the facts (i) that the $\mathrm{OH}$ stretch remains fairly decoupled from the two other degrees of freedom up to the dissociation limit, as shown by the very long dissociation time constant (in the range 10-100 ns) measured upon excitation of $\mathrm{HOCl}$ in the $\mathrm{OH}$ stretch degree of freedom, ${ }^{15-20}$ and (ii) that the $\mathrm{OH}$ bond length fluctuates only very little (between 0.9637 and 0.9725 $\AA$ ) along the minimum energy path (MEP) all the way from equilibrium to dissociation. As a consequence, the $\mathrm{OH}$ stretch degree of freedom is easily decoupled from the bend and the $\mathrm{OCl}$ stretch by freezing the $\mathrm{OH}$ bond length to its value in the free $\mathrm{OH}$ radical $(r=0.9702 \AA)$, thereby reducing the problem to a two-dimensional (2D) one. In turn, this allows for a deep insight into the vibrational dynamics of $\mathrm{HOCl}$ up to the dissociation threshold. Compared to the previous $3 \mathrm{D}$ studies, ${ }^{7,8}$ it will be shown that valuable additional information is gained with respect to the following three points.

The first point deals with POs and the bifurcations thereof. Plotting the energies of POs as a function of the Fermi polyad number (the polyad number is the total number of quanta in the coupled degrees of freedom) proved to be an essential tool for understanding the shape of the quantum wave functions of $\mathrm{HCP},{ }^{6} \mathrm{HOCl},{ }^{7}$ and $\mathrm{DCP} .{ }^{11}$ For a triatomic molecule, however, a classical analogue of the polyad quantum number can be defined rigorously for the POs of the Fermi resonance Hamiltonian, but not for those of the $a b$ initio surface. Therefore, these figures were plotted for the Fermi resonance Hamiltonian fitted against the levels of the $a b$ initio PES - not for the $a b$ initio surface itself. In contrast to the $3 \mathrm{D}$ case, it will be shown that it is possible to rigorously define a polyad number for the POs of the 2D ab initio surface. Moreover, since the dynamics is not obscured by the residual small couplings with the $\mathrm{OH}$ stretch, ${ }^{8}$ the search for the fundamental families of POs can be pursued to higher energies, so that we shall be able to address the question whether the PO born at the saddle-node bifurcation at 13000 $\mathrm{cm}^{-1}$ scars all of the dissociation states up to the threshold, or whether there exists a cascade of such bifurcations.

The second point deals with vibrational chaos. It is generally believed that the closer to the dissociation threshold, the larger the portion of phase space invaded by chaotic trajectories. Very close to the threshold one expects an essentially fully chaotic dynamics. Such an expectation seems to be reasonable for $2 \mathrm{D} \mathrm{HOCl}$, where the dissociating degree of freedom (the $\mathrm{OCl}$ stretch) is coupled to the other degree of freedom (the bend) by a strong Fermi resonance. However, the fact that the $a b$ initio spectrum can be reproduced up to $98 \%$ of the dissociation energy with an integrable Fermi resonance Hamiltonian ${ }^{7}$ suggests the opposite, i.e., that the dynamics is essentially regular. This is a question which clearly deserves further investigation, since it appears rather unlikely that chaos develops in the small energy range (the last $2 \%$ of the dissociation energy) not encompassed by the resonance Hamiltonian. It must be realized that, while classical chaos is difficult to investigate in detail in $3 \mathrm{D}$, the study of the $2 \mathrm{D}$ problem is made simple by the existence of global Poincaré surfaces of section.

The last point deals with the intramolecular vibrational energy redistribution (IVR) between the dissociating degree of freedom (the $\mathrm{OCl}$ stretch) and the other one (the bend). Conclusions regarding possible bottlenecks, that is, dynamical barriers hindering the energy from flowing between the various modes, were not expected at the beginning of this study. However, such conclusions arose naturally while plotting Poincaré surfaces of section to check the extent of vibrational chaos. It will be shown that the study of $2 \mathrm{D} \mathrm{HOCl}$ provides a clear illustration of how broken tori might act as efficient barriers preventing the spreading of energy, although the overall dynamics looks completely chaotic.

The remainder of this article is organized as follows. The principal features of the 2D $a b$ initio potential energy surfaces and the main results of the quantum calculations are summarized in Sec. II. Periodic orbits, bifurcations, and dissociation states are the focus of Sec. III, while the last section (Sec. IV) is devoted to a discussion of quantum regularity vs classical chaos and of IVR.

\section{PES AND QUANTUM STATES}

Peterson et al. ${ }^{26}$ have fitted a global PES against $a b$ initio points calculated at the multireference configuration interaction $(\mathrm{CI})$ level using large correlation-consistent basis sets. This PES was subsequently adjusted using a perturbative inversion procedure to reproduce the 22 experimentally known vibrational energies, as well as the rotational constants for nine low energy states. ${ }^{27}$ This latest version of the PES was used throughout the present work. Since the "old" surface used in the previous 3D study ${ }^{7}$ was fitted to the same ab initio data (but a smaller number of points) both PESs are of course very similar and expected to give results in good agreement. A contour plot of the 2D surface, where the $\mathrm{OH}$ bond length (the Jacobi coordinate $r$ ) is kept fixed at $r$ $=0.9702 \AA$, is displayed in Fig. 1. Horizontal and vertical axes are the Jacobi coordinate $R[R$ is the distance between the $\mathrm{Cl}$ atom and the center of mass $(G)$ of the $\mathrm{OH}$ moiety] and the cosine of the Jacobi angle $\gamma(\gamma$ is the $\mathrm{OGCl}$ angle, which is zero at the linear $\mathrm{HOCl}$ configuration), respectively. The surface is symmetric with respect to the $\gamma$ $=0$ and $\gamma=\pi$ axes. The minimum of the 2D surface [taken as the zero energy point (ZEP)] is found at $R=1.7043 \AA$ and $\gamma=1.3184 \mathrm{rad}$, that is, only $8.1 \mathrm{~cm}^{-1}$ above the absolute (3D) minimum at $R=1.7038 \AA, \gamma=1.3174 \mathrm{rad}$, and $r$ $=0.9638 \AA$. Dissociation takes place at $E=20296.3 \mathrm{~cm}^{-1}$ above the ZEP. Also indicated in the figure are the three saddle points observed below the dissociation threshold, which are due to conical intersections with excited electronic surfaces $^{21}$ and are located, respectively, at $R=1.6517 \AA$ and $\gamma=0\left(E=16105.2 \mathrm{~cm}^{-1}\right.$ above the ZEP $), R=3.4972 \AA$ and $\gamma=\pi\left(E=19840.5 \mathrm{~cm}^{-1}\right)$, and $R=3.3134 \AA$ and $\gamma=0(E$ 


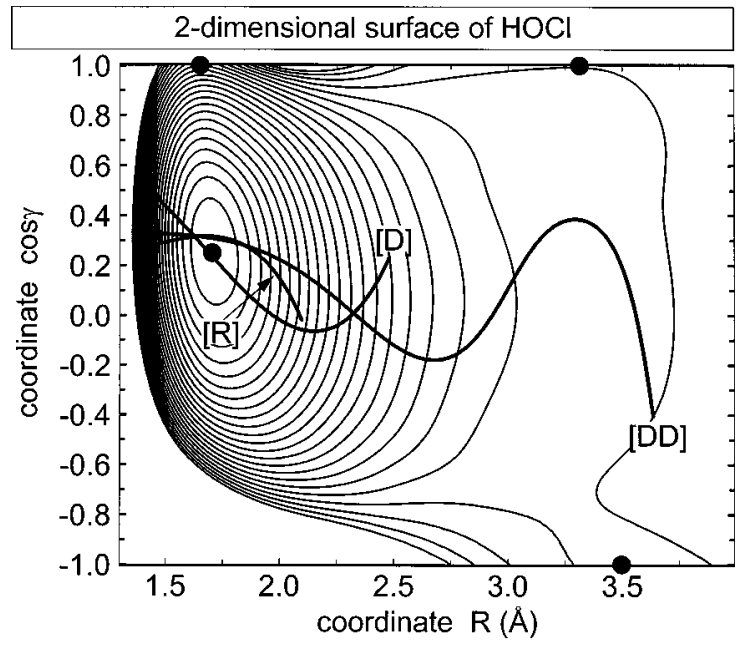

FIG. 1. Contour plot of the 2D PES of $\mathrm{HOCl}$. The $\mathrm{OH}$ bond length (the Jacobi $r$ coordinate) is kept fixed at the dissociation value $r=0.9702 \AA$. The horizontal axis is the Jacobi coordinate $R$ in Angstroms ( $R$ is the distance between the $\mathrm{Cl}$ atom and the center of mass $G$ of the $\mathrm{OH}$ moiety). The vertical axis is the cosine of the Jacobi angle $\gamma(\gamma$ is the $\mathrm{OGCl}$ angle, which is zero at the linear $\mathrm{HOCl}$ configuration). The figure is symmetric with respect to the $\gamma=0$ and $\gamma=\pi$ axes. Equipotential lines are regularly spaced between 1000 and $20000 \mathrm{~cm}^{-1}$ above the minimum, with increments of $1000 \mathrm{~cm}^{-1}$. The black dots indicate the positions of the minimum and the three saddle points. Also plotted are the $[R],[D]$, and $[D D]$ POs at 6500 , 14000 , and $19000 \mathrm{~cm}^{-1}$ above the ground state, respectively. These are the three families of stable POs, which successively scar the dissociation states (see text).

$=20011.9 \mathrm{~cm}^{-1}$ ). Finally, let us mention for completeness that $\mathrm{HOCl}$ has an isomer, $\mathrm{HClO}$, below the dissociation energy to $\mathrm{OH}+\mathrm{Cl}$ and that quantum calculations have been performed for this isomer. ${ }^{26}$ The barrier separating both isomers is however above the dissociation threshold of $\mathrm{HClO}$ and it is too high and broad for $\mathrm{HClO}$ to play any role in the dissociation of $\mathrm{HOCl}$.

Vibrational variational calculations were performed using a truncation/recoupling method described in detail in Ref. 22. The basis used for constructing the 2D Hamiltonian matrix consists of the direct product of $2201 \mathrm{D}$ radial functions extending from 2 to $15 \mathrm{bohr}$ and $601 \mathrm{D}$ angular functions. The 1D functions were obtained by diagonalization of suitable 1D Hamiltonians in discrete variable representation. Resonance widths/dissociation rates for 2D states above dissociation threshold (end of Sec. III B) were computed using the complex L2 method with optical potential. ${ }^{23}$ The 2D quantum ground state is found at $E=987.2 \mathrm{~cm}^{-1}$ above the ZEP. Except when explicitly noted, all the energies in the remainder of this article are given relative to the quantum ground state, in order to facilitate the comparison with the $3 \mathrm{D}$ results. The wave functions of all the 355 bound states, as well as the first 40 resonances up to $175 \mathrm{~cm}^{-1}$ above the threshold, were examined visually and assigned a quantum polyad number $P$. The energy range, in which the wave functions can be uniquely assigned, is thus considerably larger than in the 3D studies, where the assignment procedure had to be stopped about $400 \mathrm{~cm}^{-1}$ below the threshold. Let us recall that $P$ is an approximate quantum number for $a b$ initio states, which is defined according to

$$
p=2 v_{2}+v_{3},
$$

where $v_{2}$ and $v_{3}$ denote the number of quanta in the bend and $\mathrm{OCl}$ stretch degrees of freedom, respectively. Use of $P$, instead of the more usual $v_{2}$ and $v_{3}$ quantum numbers, is made necessary by the Fermi resonance, which couples the $\mathrm{OCl}$ stretch and the bend degrees of freedom $\left(\omega_{2} \approx 2 \omega_{3}\right)$. Let us also mention that the bound state with highest number of quanta in the $\mathrm{OCl}$ stretch is no. 354, which is located only $11.9 \mathrm{~cm}^{-1}$ below the threshold and is the lowest member of polyad $P=46$.

An example of how well the states of the 2D surface compare with those of the full 3D calculation is provided in Fig. 2. This figure shows the wave functions in the $[R, \cos (\gamma)]$ plane for the 16 states belonging to polyad $P$ $=30$ and compares readily with Fig. 4 of Ref. 7 and Fig. 9 of Ref. 8. It is seen that these three figures are rather similar, the principal difference being that the states with clear bending character are the 3rd and 5th lowest ones according to the 3D surface, but the 7th and 9th lowest ones according to the 2D surface. This difference merely reflects the fact that the energy of the families of bending-type POs varies somewhat inside a given polyad when going from $3 \mathrm{D}$ to $2 \mathrm{D}$. This is, however, of little consequence since the majority of quantum states is scarred by the other families of stable periodic orbits (cf. next section).

As for the 3D study, ${ }^{7}$ the $a b$ initio spectrum could be reproduced up to $98 \%$ of the dissociation energy with a Fermi resonance Hamiltonian: the first 320 bound states (with up to 40 quanta in the $\mathrm{OCl}$ stretch) were fitted with an effective Hamiltonian containing 32 parameters, leading to rms and maximum errors of 4.8 and $19.9 \mathrm{~cm}^{-1}$, respectively. In addition to these 320 bound states, most higher levels are also well predicted by the Fermi resonance Hamiltonian, the exceptions being the states extending along the dissociation pathway. It was furthermore checked that the wave functions of the 2D Fermi resonance Hamiltonian compare well with the 2D ab initio ones, just as in Figs. 4 and 5 of Ref. 7. The discussion concerning chaos in Sec. IV A relies on the existence of this integrable resonance Hamiltonian. In contrast with the 3D calculations, however, the detailed study of the 2D resonance Hamiltonian does not yield new information compared to the 2D study employing the $a b$ initio $2 \mathrm{D}$ surface. Therefore, the 32 fitted parameters are not given here.

\section{PERIODIC ORBITS, BIFURCATIONS, AND DISSOCIATION STATES}

In this section, we first recall what a "fundamental family" of POs is and we show how a classical version of the quantum polyad number can be defined for such POs. We then study the bifurcation diagram for these POs in connection with the shape of the quantum wave functions, particularly emphasising the so-called dissociation states, which closely follow the dissociation pathway.

\section{A. Fundamental families of POs: Definition of a classical polyad number}

The POs of the 2D ab initio surface were located using two-point boundary value solvers, as described in Sec. II of 


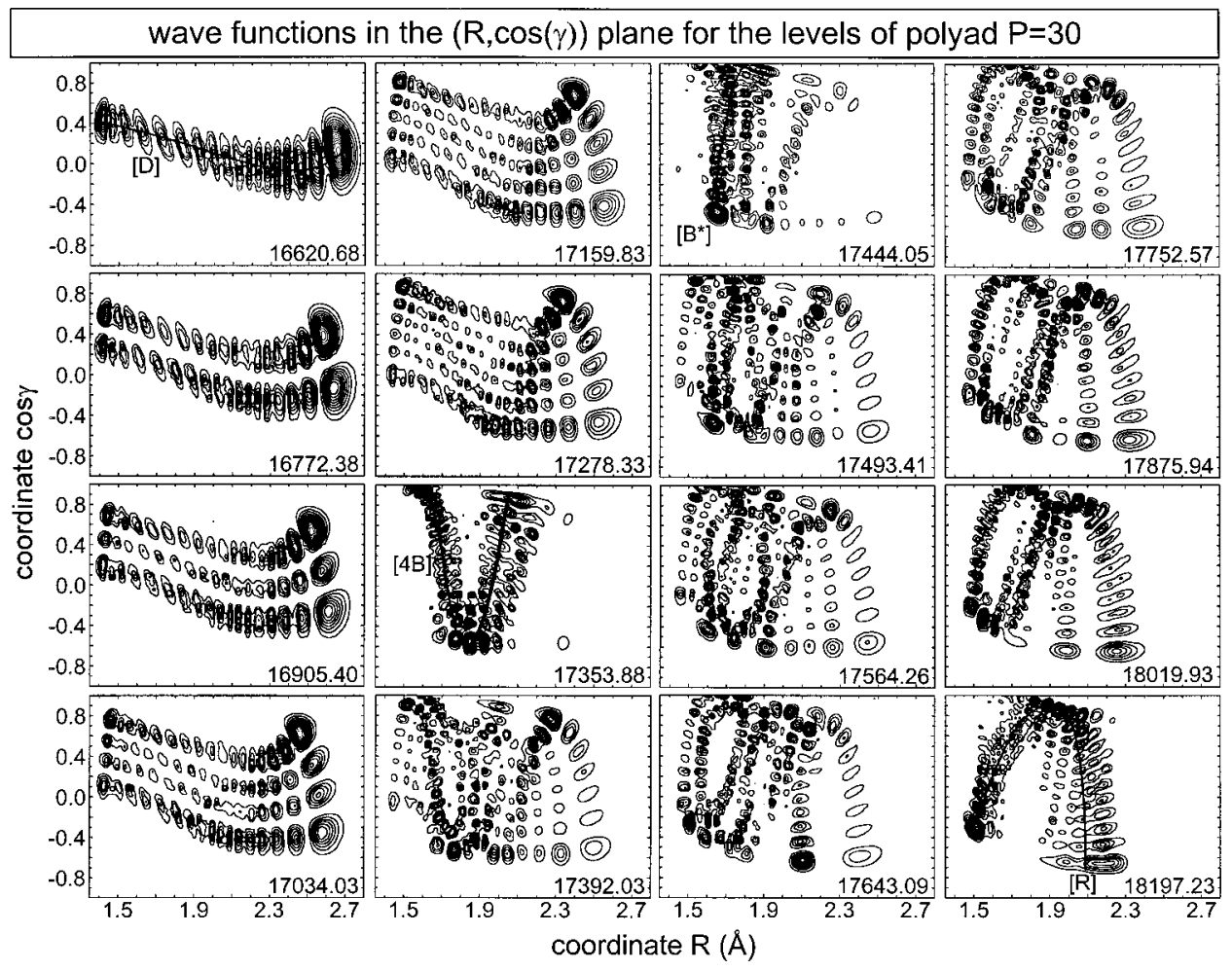

FIG. 2. Wave functions in the $[R, \cos (\gamma)]$ plane for the 16 states belonging to polyad $P=30$, according to 2D calculations. This figure compares readily with Fig. 4 of Ref. 7 and Fig. 9 of Ref. 8, which display the same wave functions obtained, however, from 3D calculations. The energy relative to the quantum ground state is indicated for each wave function. Also shown on top of the corresponding wave functions are the $[D]$ stable PO, which scars the lowest quantum state, the $[4 B]$ stable PO, which scars the 7 th lowest state, the $\left[B^{*}\right]$ unstable PO, which scars the 9th lowest state, and the $[R]$ stable $\mathrm{PO}$, which scars the highest state.
Ref. 30. It is to be noted that only principal families of POs were sought for, that is, those families of POs which really correspond to distinct molecular motions. Excluded from the search were the POs arising from the destruction of rational tori due to nonintegrable couplings (that is, all the couplings besides Fermi resonance) and their replacement by families of stable and unstable POs. ${ }^{31,32}$ The reason for discarding these latter POs, which do scar some quantum wave functions (see Figs. 6 to 10 of Ref. 31), is that they cause the bifurcation diagram to become very complex, without providing much additional insight into the molecular dynamics. Indeed, POs born from the destruction of rational tori are merely linear combinations of the fundamental families of POs (see, for example, Figs. 1 and 2 of Ref. 31) and quantum wave functions scarred by such POs can often be described in terms of the principal families of POs. For example, the second highest state of polyad $P=30$ ( $E$ $=18019.93 \mathrm{~cm}^{-1}$ ), shown in Fig. 2, can be thought of either as a state scarred by a PO born from the destruction of a 1:2 rational torus, or as a state displaying one node perpendicular to the $[R] \mathrm{PO}$.

At this point, it is worth mentioning that it is not always easy to distinguish, from the numerical point of view, between POs arising from the destruction of rational tori and fundamental families of POs born at a saddle-node bifurcation. Nonetheless, the examination of the shape of the PO might give a sound indication regarding its origin. For example, some POs which are reported in the bifurcation diagram of the 3D ab initio surface of $\mathrm{HOCl}$ (Fig. 5 of Ref. 8) have little influence on quantum wave functions. Since these POs do not appear in the bifurcation diagram for the corresponding 3D Fermi resonance Hamiltonian (Fig. 7 of Ref. 7), the suspicion naturally arises that these additional POs might originate from the destruction of rational tori. A first example is the PO labeled $[\gamma]$ shown in Fig. 11(a) and 11(b) of Ref. 8. According to this figure, $[\gamma]$ is clearly the linear combination of one time $[\tilde{\gamma}]$ (the fundamental PO along the bend degree of freedom) plus three times $[r]$ (the fundamental PO along the $\mathrm{OH}$ stretch degree of freedom). Therefore, $[\gamma]$ probably originates from the destruction of the degenerate $2 \mathrm{D}$ rational torus ${ }^{33}$ with no motion along the $\mathrm{OCl}$ stretch and a 3:1 rational ratio between the classical frequencies associated with the $\mathrm{OH}$ stretch and the bend degrees of freedom. Similarly, the $[R] \mathrm{PO}$ shown in Figs. 11(c) and 11(d) of Ref. 8 probably originates from the destruction of the degenerate 2D torus with no motion along the bend and a 5:1 rational ratio between the classical frequencies associated with the $\mathrm{OH}$ and $\mathrm{OCl}$ stretches. Although the cascade of POs labeled $\left[\mathbf{D}_{i}\right]$ in Fig. 5 of Ref. 8 are not plotted in configuration space, it is plausible that they also originate from the destruction of degenerate $2 \mathrm{D}$ rational tori with no motion along the bending coordinate. If one withdraws these POs from the bifurcation diagram of the 3D ab initio surface (Fig. 5 of Ref. 8), then it displays just the same POs as the bifurcation diagram of the 3D Fermi resonance Hamiltonian (Fig. 7 of Ref. 7), which looks simpler although it contains all the necessary information for understanding the shape of quantum wave functions.

As stated in the introduction, a classical version of the quantum polyad number $P$ is easily derived for each PO of the 2D ab initio surface. Indeed, as shown by a comparison with the Fermi resonance Hamiltonian, for which the quantum polyad number $P$ and its classical counterpart can be defined exactly, ${ }^{7} P$ is simply proportional, in $2 \mathrm{D}$ systems, to the total action integral along the PO: 


$$
P=2^{-N} \frac{1}{2 \pi \hbar} \oint_{P O} p_{R} d R+p_{\gamma} d \gamma-\frac{3}{2} .
$$

$N$ depends on whether the PO is born as an essentially bend $(N=-1)$ or $\mathrm{OCl}$ stretch $(N=0)$ motion and increases by one each time the PO undergoes a period-doubling bifurcation. More precisely, $N$ is equal to -1 for the POs labeled $[B]$ and $\left[B^{*}\right]$ in the remainder of this article, to 0 for the POs labeled $[R],[2 B],[D],\left[D^{*}\right]$, and $[D D]$, and to 1 for the POs labeled $[2 R],[4 B]$, and $[2 D]$. The $3 / 2$ term is the sum of Maslov indexes and must be substracted according to the Einstein-Brillouin-Keller (EBK) semiclassical quantization rules. ${ }^{34-36}$

\section{B. Bifurcation diagram, "inversion" and "dissociation" states}

Using Eq. (3.1), one is able to plot the energies of the POs as a function of the (classical) polyad number $P$. Because of the Fermi resonance, however, the energies of all the POs are almost degenerate and the resulting plot is unclear. A better solution therefore consists in plotting the energies of the POs relative to the energy of one of the POs or to some other reference, which stays close to the bottom of the polyad. In the $3 \mathrm{D}$ study, ${ }^{7}$ the reference was taken as the energy of the pure bend PO of the Fermi resonance Hamiltonian. This choice is no longer convenient here, because the studied polyad range extends up to $P=46$, instead of $P$ $=38$ for the $3 \mathrm{D}$ study, and the separation between the pure bend PO and the bottom of the polyad increases (because of the very strong anharmonicity of the dissociation states) by several thousands of $\mathrm{cm}^{-1}$ between $P=38$ and $P=46$. A better reference for the present study is found to be

$$
\begin{aligned}
E_{a v}(P)= & 114.59+728.038 P-5.61245 P^{2} \\
& -0.0324305 P^{3} \\
& +0.00255035 P^{4}-0.0000447972 P^{5},
\end{aligned}
$$

when expressed in $\mathrm{cm}^{-1}$ above the ground state. The plot of $E_{\text {av }}$ as a function of $P$ is given in the small insert of Fig. 3. The main part of Fig. 3 shows the energies of the POs relative to $E_{\text {av }}$ as a function of $P$. Solid lines denote stable POs and doted lines unstable ones. The large black dots labeled (a) to (f) indicate the bifurcations and will be discussed below. It is worth emphasizing again that, for each value of $P$, the classically accessible region, as well as the quantum states belonging to polyad $P$, are always bordered by the two outermost stable POs. For the sake of illustration, the 16 levels belonging to polyad $P=30$ are shown as a column of small black dots. The quantum state nearest to a PO is generally "scarred" by this particular PO, that is, the PO acts as a "necklace", on which the "pearls" of the quantum wave function are threaded.

Let us now discuss in detail the principal families of POs and their bifurcations. The PO, which coincides with the $\mathrm{OCl}$ stretch normal mode close to the ZEP, is called $[R]$. This is the PO with highest energy throughout the investigated polyad range. Therefore, it is expected to scar the highest state in each polyad, as can be verified in Fig. 2, where the $[R] \mathrm{PO}$ is plotted on top of the wave function of the highest

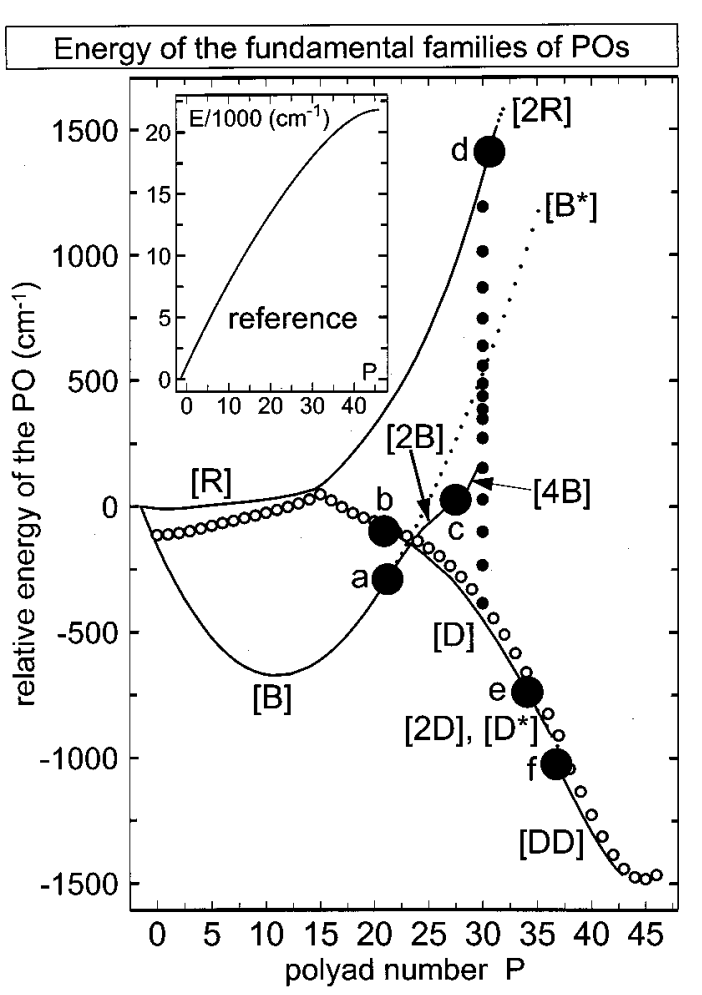

FIG. 3. (Small inset) plot of the reference energy $E_{\text {av }}$ in Eq. (3.2) as a function of $P$. (Overall figure) plot of the energy of the POs relative to $E_{\mathrm{av}}$ as a function of the classical polyad number $P$ defined in Eq. (3.1). Solid and dotted lines stand for stable and unstable POs, respectively. Large black dots labeled (a) to (f) indicate where bifurcations take place. The 16 levels belonging to the quantum polyad $P=30$ are shown as a column of small black dots and the 47 dissociation states from $P=0$ to $P=46$ as a line of small open circles.

state of polyad $P=30$. This $P O$ follows the dissociation pathway up to approximately $P=15$ (about $10000 \mathrm{~cm}^{-1}$ above the ground state). For higher polyads, however, $[R]$ progressively acquires a pronounced "horseshoe" shape, thereby avoiding the dissociation pathway. This evolution is very clear upon comparison of Figs. 1 and 2, where $[R]$ is plotted at $6500 \mathrm{~cm}^{-1}$ and $18200 \mathrm{~cm}^{-1}$, respectively. This behavior is in good agreement with the $3 \mathrm{D}$ results. ${ }^{7,8}$ The present 2D study further points out a period-doubling bifurcation [the black dot labeled (d) in Fig. 3], which takes place around $P=30.6$ and $E=18600 \mathrm{~cm}^{-1}$. The stable PO born at this bifurcation, which is called $[2 R]$ to remind that it essentially consists of the $[R] \mathrm{PO}$ covered twice, becomes itself unstable only about $200 \mathrm{~cm}^{-1}$ above the bifurcation. In contrast, the unstable $[R] \mathrm{PO}$, which is not shown in Fig. 3, could be followed up to $P=43$ and $E=25000 \mathrm{~cm}^{-1}$, that is, largely above the dissociation threshold. Although chaos will be studied more specifically in the next section, let us mention that this period-doubling bifurcation and the early destabilization of $[2 R]$ reveal that the remaining small island of regular motion centered around $[R]$ is completely and definitely destroyed by chaos. Perhaps, there exists a whole cascade of such period-doubling bifurcations, the regular islands becoming, however, smaller and smaller and escaping careful numerical research.

The PO, which coincides with the bend normal mode 
close to the ZEP, is called $[B]$. As in the 3D case, it undergoes a period-doubling bifurcation around $P=21$ and $E$ $=12500 \mathrm{~cm}^{-1}$. This is the black dot labeled (a) in Fig. 3 . The bend PO is unstable above the period-doubling bifurcation and is called $\left[B^{*}\right]$ to emphasize this point. $\left[B^{*}\right]$ is up to 17000 or $18000 \mathrm{~cm}^{-1}$, however, only slightly unstable, so that some quantum states are scarred by $\left[B^{*}\right]$ up to this energy regime. This is clearly seen in Fig. 2, where the $\left[B^{*}\right] \mathrm{PO}$ is plotted on top of the wave function of the 9 th lowest quantum state of polyad $P=30$, which is located at about $17450 \mathrm{~cm}^{-1}$. The stable PO born at the bifurcation is called $[2 B]$ and displays, like $[R]$, a horseshoe shape in the $[R, \cos (\gamma)]$ plane. While $[B]$ remains the PO with the lowest energy inside a given polyad up to this bifurcation, $[2 B]$ and $\left[B^{*}\right]$ penetrate the core of the polyad shortly above the bifurcation and are subsequently replaced by the $[D] \mathrm{PO}$ (to be discussed in the following paragraphs) at the bottom of the polyad. This is the reason, why states scarred by bendingtype POs are the bottom ones in each polyad below $P=24$ and then move higher in the polyad, i.e., the $[D]$ states fall below the bending states, because of their large anharmonicity. This is again in good agreement with the 3D calculations. ${ }^{7,8}$ The examination of the $2 \mathrm{D}$ dynamics shows that $[2 B]$ further undergoes a second period-doubling bifurcation at about $P=27$ and $E=15500 \mathrm{~cm}^{-1}$, corresponding to the black dot labeled (c) in Fig. 3. At this energy, $[2 B]$ crosses the $\gamma=0$ axis close to the saddle-point with lowest energy and penetrates the equivalent well with negative values of $\gamma$. The motion along the stable PO born at this bifurcation (which is called $[4 B]$ ) consists of a $[2 B] \mathrm{PO}$ in the well with $\gamma>0$ followed by a second $[2 B] \mathrm{PO}$ in the well with $\gamma<0$. If one realizes that the $[4 B] \mathrm{PO}$ connects two equivalent equilibrium positions through the linear $\mathrm{HOCl}$ unstable configuration, then it is clear that quantum states scarred by $[4 B]$ should be called "inversion" states. The 7 th lowest state of polyad $P=30$ shown in Fig. 2 is an example of such an inversion state. However, the number of inversion states in the spectrum of $\mathrm{HOCl}$ is limited to a few (4 or 5), since the $[4 B] \mathrm{PO}$ becomes unstable very soon above the bifurcation, around $P=30$ and $E=17400 \mathrm{~cm}^{-1}$. Here again, the destabilization of the $[4 B] \mathrm{PO}$ indicates that the last island of regular motion around $[4 B]$ is being definitely destroyed by chaos.

As in the 3D calculations, a first saddle-node bifurcation is found to take place around $P=21$ and $E=12800 \mathrm{~cm}^{-1}$. This is the black point labeled (b) in Fig. 3. The stable PO born at this bifurcation is called $[D]$, because it follows the dissociation pathway in a broad energy range. Very rapidly, around $P=23,[D]$ becomes the lowest limit of the accessible energy range and is therefore expected to scar the wave function of the state with lowest energy in each polyad. This can be verified in Fig. 2, where the $[D] \mathrm{PO}$ is plotted on top of the wave function of the lowest state of polyad $P=30$. Quantum states scarred by $[D]$ were called dissociation states in the $3 \mathrm{D}$ work, ${ }^{8}$ in order to emphasize their role in the dissociation dynamics. In the $2 \mathrm{D}$ system, $[D]$ further undergoes a period-doubling bifurcation close to $P=34$ and $E$ $=17500 \mathrm{~cm}^{-1}$. This is the black point labeled (e) in Fig. 3 . The stable PO born at this bifurcation, which for obvious

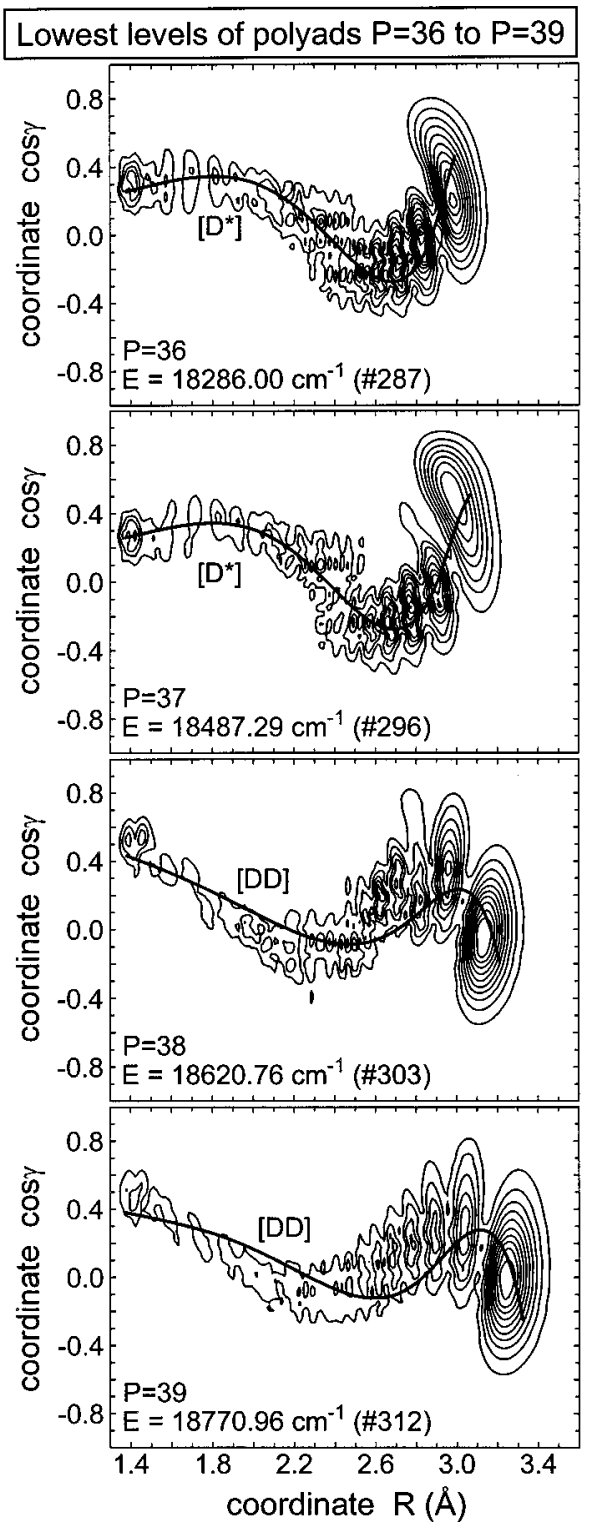

FIG. 4. Wave functions in the $[R, \cos (\gamma)]$ plane for the lowest state of polyads $P=36$ to $P=39$. Plotted on top of the wave functions are the unstable $\left[D^{*}\right] \mathrm{PO}$ and the stable $[D D] \mathrm{PO}$.

reasons is called $[2 D]$, becomes in turn unstable shortly above the bifurcation, slightly below $P=37$ and $E$ $=18500 \mathrm{~cm}^{-1}$. The reason for this period-doubling bifurcation and the early destabilization of $[2 D]$ is once more the chaotic invasion of the last island of regular motion around $[D]$. The unstable $[D] \mathrm{PO}$ (called $\left[D^{*}\right]$ ) remains slightly unstable up to about $18400 \mathrm{~cm}^{-1}$, while the Lyapunov exponent increases dramatically for higher energies.

The investigation of the quantum/classical correspondence through the study of fundamental families of POs is however still not finished. Indeed, looking at the wave functions of the lowest state of polyads $P=36$ to $P=39$, which are reproduced in Fig. 4, it appears that something new necessarily happens between polyads $P=37$ and $P=38$ : In contrast with the lowest levels of polyads $P=36$ and $P=37$, which are clearly scarred by $\left[D^{*}\right]$, the nodal lines of the wave functions of the lowest levels of polyads $P=38$ and 
$P=39$, as well as higher polyads (see, for example, Fig. 7), clearly do not resemble the $\left[D^{*}\right] \mathrm{PO}$, although they stretch along the dissociation pathway. This figure suggests that a second saddle-node bifurcation occurs in this polyad range for the 2D system. This additional saddle-node bifurcation is actually found around $P=36.5$ and $E=18300 \mathrm{~cm}^{-1}$. It is indicated with the black dot labeled (f) in Fig. 3. The stable PO born at this bifurcation, which is called $[D D]$, remains stable (or only very slightly unstable) up to $P \approx 43$ and $E$ $=19100 \mathrm{~cm}^{-1}$, that is, less than $200 \mathrm{~cm}^{-1}$ below the dissociation threshold, and replaces $[D],\left[D^{*}\right]$, and $[2 D]$ at the low energy end of the polyads.

If one defines dissociation states in a more general way, as being those states for which all the energy flows along the dissociation pathway, then their behavior can be summarized as follows.

(1) Up to $P=15$, dissociation states are the highest level in each polyad and are scarred by the $[R] \mathrm{PO}$.

(2) Between polyads $P=16$ and $P=23$, dissociation states move from the top to the bottom of the polyads. They often mix strongly with other states and are not scarred by any particular PO, except for the states belonging to polyads $P=22$ and $P=23$, which are scarred by $[D]$.

(3) Between $P=24$ and $P=37$, dissociation states are the lowest level in each polyad and are scarred by $[D]$ (up to $P=34)$ and $\left[D^{*}\right](P=35$ to $P=37)$.

(4) Between $P=38$ and $P=43$, dissociation states are the lowest level in each polyad and are scarred by $[D D]$.

(5) the remaining three bound dissociation states (belonging to polyads $P=44$ to $P=46$ ) are the lowest level in each polyad. Superposition of their wave functions with the $[D D] \mathrm{PO}$ at $19100 \mathrm{~cm}^{-1}$ shows that these states are not scarred by $[D D]$, but more likely by another family (or several families) of POs, which are born at saddle-node bifurcations(s) around or above $P=43$ (in the last $200 \mathrm{~cm}^{-1}$ below the dissociation threshold) and which stretch along the dissociation pathway up to larger and larger values of $R$. However, we were unable to find these additional bifurcations.

(6) Dissociation states can even be followed above the dissociation threshold. Not surprisingly, they correspond to the resonances with shortest lifetime, as can be expected from the fact that all the energy flows along the dissociation pathway. For example, the third and fourth resonances computed above the threshold, which can be assigned as the lowest "levels" of polyads $P=47$ and $P=48$, respectively, have computed lifetimes about one million times shorter than the fifth resonance, which is assigned as the 10th lowest state of polyad $P=34$ (see also the discussion in Refs. 8, 25, 28).

The evolution of dissociation states is illustrated in Figs. 1 and 3: The three families of POs which scar dissociation states, namely $[R],[D]$, and $[D D]$, are plotted in Fig. 1 on top of the contour plot of the PES at energies of 6500, 14000 , and $19000 \mathrm{~cm}^{-1}$, respectively. This figure shows how each PO follows the dissociation pathway in a given energy range before curving and escaping it. All the dissociation states from the ground state $(P=0)$ up to the highest bound dissociation state $(P=46)$ are further indicated with small open circles in Fig. 3. The agreement between quantum results (open circles) and classical ones obtained from
Eq. (3.1) (solid lines) is seen to be perfect, except at very low energies, where the notion of dissociation states is not well founded.

\section{VIBRATIONAL CHAOS AND IVR}

In this section we first show, through the comparison of classical Poincaré surfaces of section (SOSs) and quantum Husimi distributions, that the classical dynamics of $2 \mathrm{D} \mathrm{HOCl}$ looks much more chaotic than the quantum dynamics. This observation is discussed in terms of the quantum/classical correspondence. We then show that quantum and classical mechanics nevertheless agree in predicting a slow intramolecular vibrational energy redistribution (IVR) between dissociation and nondissociation motions.

\section{A. Quantum regularity vs. classical chaos}

Poincaré surfaces of section (SOSs) are a powerful tool for studying chaos in 2D classical mechanics. The SOSs drawn in the left column of Fig. 5 consist of the points $\left(p_{\gamma}, \gamma\right)$ taken along several trajectories at those times for which $R$ is equal to $1.70 \AA$ and $p_{R}$ is positive. The energy $E$ relative to the quantum mechanical ground state is equal to 14000, 17000 , and $18500 \mathrm{~cm}^{-1}$ for the top, middle, and bottom plots, respectively. The SOSs obtained from the same trajectories, but computed at $\gamma=1.32 \mathrm{rad}$ and $p_{\gamma}>0$ and plotted in the $\left(p_{R}, R\right)$ plane, are displayed in the right column of the same figure. Regular (nonchaotic) dynamics is signified when the SOS cuts the motion in one or several closed curves, since this implies that the motion is located on a torus. In contrast, areas filled with random-looking arrays of points indicate that tori no longer exist in these regions and that the corresponding dynamics is therefore chaotic.

Examination of the SOSs at $E=14000 \mathrm{~cm}^{-1}$ shows that the two lowest bifurcations [points (a) and (b) in Fig. 3], which take place at about 12500 and $12800 \mathrm{~cm}^{-1}$, respectively, play a fundamental role in the appearance of vibrational chaos, since the first macroscopic region filled with chaotic trajectories clearly develops around the unstable POs created at these bifurcations. Large islands of regular (or almost regular) motion however still exist around each one of the stable POs, namely $[R],[2 B]$ and $[D]$. At $E$ $=17000 \mathrm{~cm}^{-1}$, the bend PO [which is called $[4 B]$ above the (c) bifurcation at $\left.15500 \mathrm{~cm}^{-1}\right]$ can still be found, but the island of regular motion around it has almost totally vanished; it is reminded that $[4 B]$ itself becomes unstable around $17400 \mathrm{~cm}^{-1}$. The size of the regular island around the $[R] \mathrm{PO}$ has also strongly decreased, while the size of the island around $[D]$ remains mostly unchanged compared to $E=14000 \mathrm{~cm}^{-1}$. At $E=18500 \mathrm{~cm}^{-1}$, that is, about 800 $\mathrm{cm}^{-1}$ below the dissociation threshold, all of the regular regions have disappeared, except for a very thin crescent around $[R]$. This last crescent vanishes about $300 \mathrm{~cm}^{-1}$ higher in energy and Poincaré SOSs looks henceforth totally chaotic.

The finding that chaos spreads over all the phase space at these energies contrasts with the obvious regularity of many of the $a b$ initio quantum wave functions (cf. Fig. 2) and the ability to reproduce the $a b$ initio spectrum with a fully integrable (i.e., nonchaotic) Fermi resonance Hamiltonian up to 


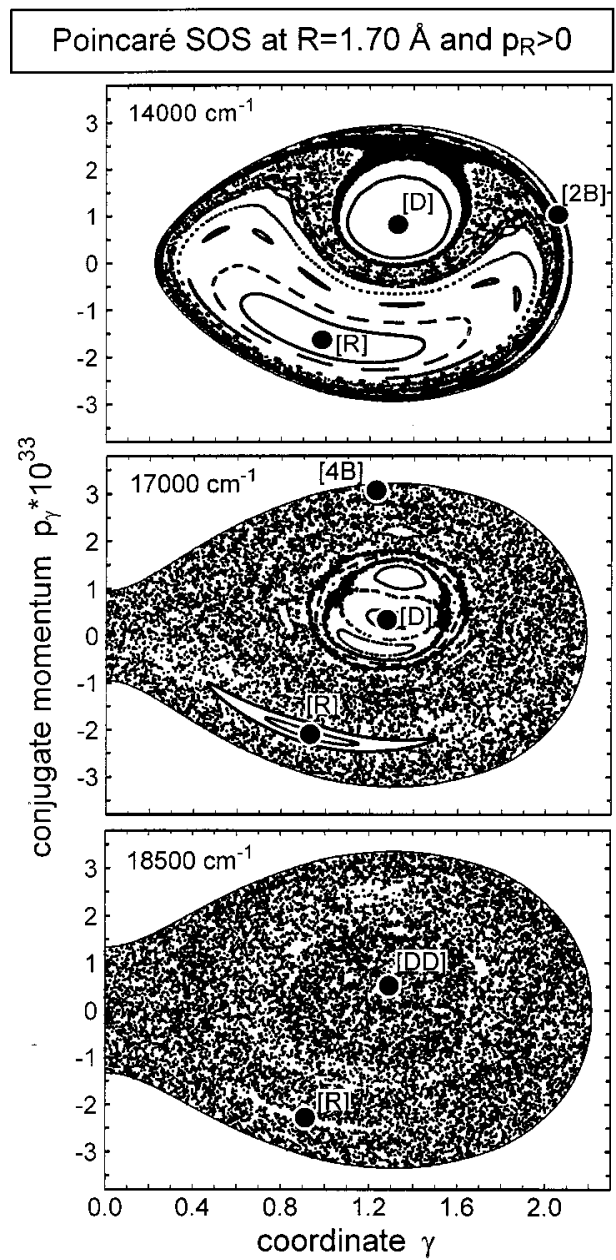

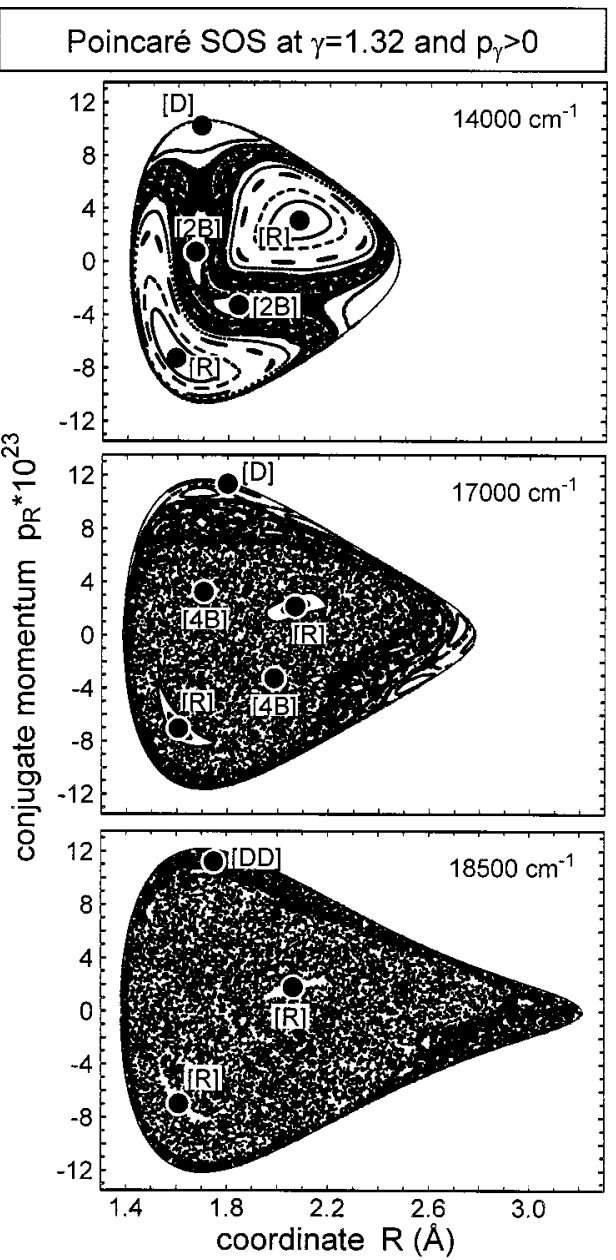

FIG. 5. The left column displays Poincaré surfaces of section in the $\left(\gamma, p_{\gamma}\right)$ plane at $R=1.70 \AA$ and the right column the corresponding SOSs in the $\left(R, p_{R}\right)$ plane at $\gamma=1.32 \mathrm{rad}$. The energy increases from $14000 \mathrm{~cm}^{-1}$ above the quantum mechanical ground state (top) to $17000 \mathrm{~cm}^{-1}$ (middle) and $18500 \mathrm{~cm}^{-1}$ (bottom). The large black dots indicate where the stable POs cross the surfaces of section.
$19900 \mathrm{~cm}^{-1}$, that is, $400 \mathrm{~cm}^{-1}$ below the dissociation threshold. In order to make this discrepancy between quantum and classical mechanics more obvious, we plotted in Fig. 6 the Husimi distributions ${ }^{37-40}$ for the 16 states belonging to polyad $P=30$, whose quantum wave functions are displayed in Fig. 2. The Husimi distribution $\rho$ for a given wave function $\Psi$ is a function of the coordinates $\mathbf{q}=(R, \gamma)$ and $\mathbf{p}=\left(p_{R}, p_{\gamma}\right)$, such that ${ }^{37-40}$

$$
\rho(\Psi ; \mathbf{p}, \mathbf{q})=\frac{1}{(2 \pi \hbar)^{2}}\left|\left\langle\Phi_{\mathbf{p}, \mathbf{q}}, \Psi\right\rangle\right|^{2} .
$$

In this equation, $\Phi_{\mathbf{p}, \mathbf{q}}$ is the minimum uncertainty wave packet centered at $(\mathbf{q}, \mathbf{p})$, that is, it is the product of two 1D functions of the form

$$
\begin{aligned}
\Phi_{p, q}^{1 D}\left(q^{\prime}\right)= & \frac{1}{\left(2 \pi(\Delta q)^{2}\right)^{1 / 4}} \\
& \times \exp \left\{-\frac{1}{4(\Delta q)^{2}}\left(q^{\prime}-q\right)^{2}+\frac{i}{\hbar} p\left(q^{\prime}-q\right)\right\} .
\end{aligned}
$$

The $\Delta q$ s are the half-widths of the 1D minimum uncertainty wave packets, which are estimated according to

$$
\Delta q=\sqrt{\frac{\hbar}{2}}\left(\frac{\partial^{2} T}{\partial p^{2}}\right)^{1 / 4}\left(\frac{\partial V}{\partial q^{2}}\right)^{-1 / 4}
$$

For $\mathrm{HOCl}$, one finds $\Delta R=0.044 \AA$ and $\Delta \gamma=0.050 \mathrm{rad}$. Figure 6 shows contour plots in the $\left(\gamma, p_{\gamma}\right)$ plane of the Husimi distributions for the 16 states of polyad $P=30$, where $R$ is fixed at $1.70 \AA$ and $p_{R}$ is chosen such that the total energy $E$ is equal to the energy of the corresponding quantum state. Each plot of Fig. 6 is therefore just the quantum equivalent of a classical Poincaré SOS drawn for a given quantum state, which is the reason, why such plots are also known as " "quantum Poincaré maps.', 39 Not surprisingly, Husimi distributions agree with wave functions in that they display a very regular behavior: the six lowest states of the polyad are dissociation states and their Husimi distributions are accordingly localized inside the "pit" of the "avocado," which surrounds the $[D] \mathrm{PO}$; the next three states have a primarily bending nature and $\rho$ is rightly localized on the circumference of the "avocado," along which trajectories close to $[2 B]$ and $[4 B]$ do loop; at last, the seven states with highest energy are scarred by $[R]$ and $\rho$ is seen to be maximum in the crescent surrounding $[R]$. For the states scarred by $[D]$ and $[R]$ one can even count the number of nodes in the Husimi distributions in order to find the rank of the state in the polyad. It is worth noting how different the distributions in Fig. 6 look compared to the chaotic ones shown in Fig. 2 of Ref. 39. Indeed, the latter ones spread over the whole accessible phase space and display very irregular nodal lines. 


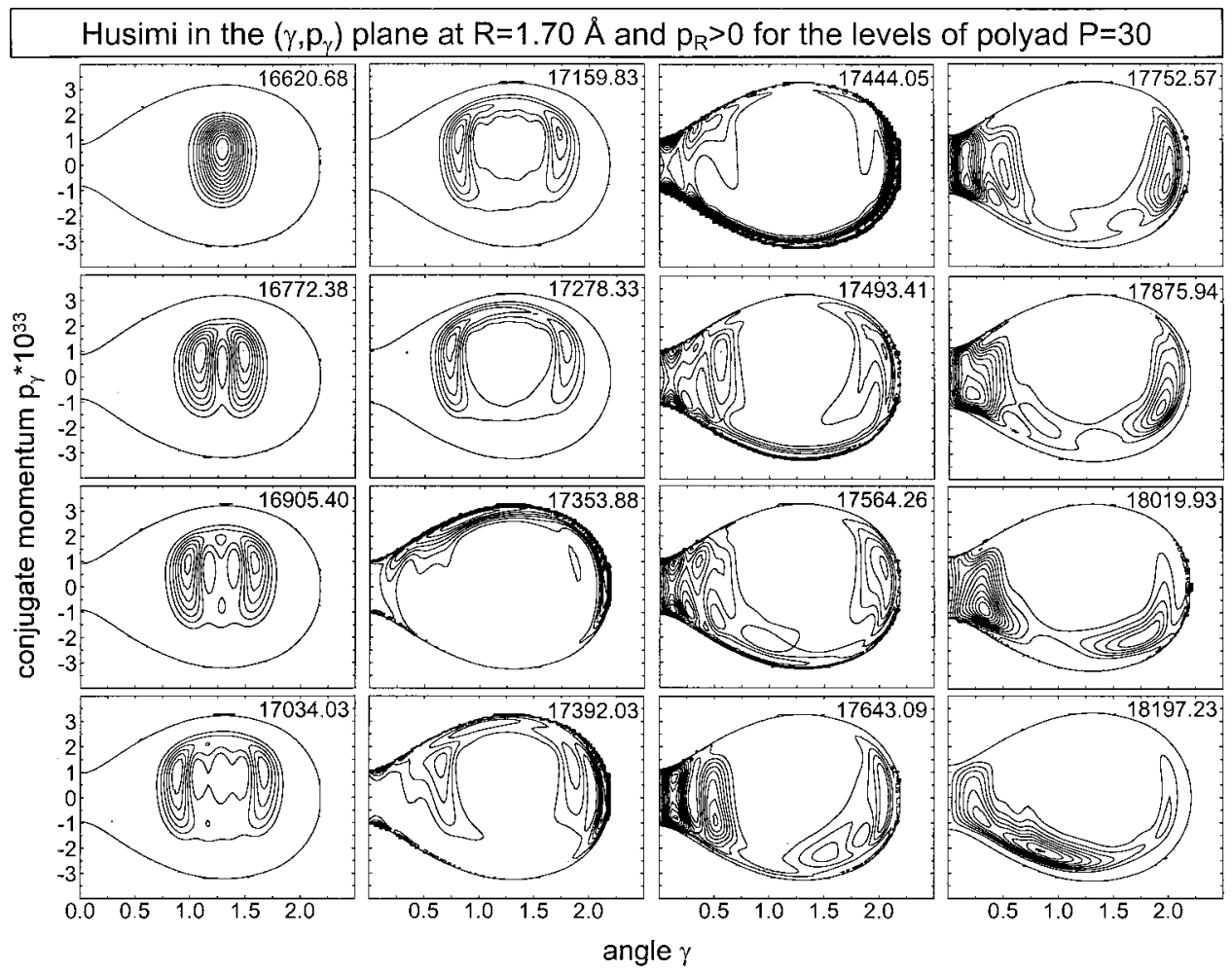

FIG. 6. Plot of Husimi distributions in the $\left(\gamma, p_{\gamma}\right)$ plane for the 16 levels belonging to polyad $P=16$, whose wave functions are shown in Fig. 2. $R$ is kept fixed to the equilibrium value $(R=1.70 \AA)$ and $p_{R}$ is chosen such that the total energy be equal to the energy of each quantum state. Husimi distributions plotted this way are just the quantum analogues of Poincaré SOSs plotted in the left column of Fig. 5.
Although striking, the fact that classical dynamics looks much more chaotic than quantum dynamics is not new and has been discussed in some detail by Davis, ${ }^{41,42}$ Reinhardt and co-workers, ${ }^{43-48}$ and Eckardt et al $^{49}$ According to Reinhardt and co-workers, who studied the Henon-Heiles surface, this is due to the fact that quantizing tori would not be completely destroyed by nonlinear couplings, but considerable "structure" (local constants of motion) would instead survive. This structure would be able to confine trajectories on a torus for many vibrational periods, except at some places, where the motion would be highly complex on the scale of one (or less than one) vibrational period. In these latter regions of complexity, nearby trajectories would separate exponentially and transitions between remnants of tori would take place. This point is illustrated in Fig. 6 of Ref. 45 and Fig. 9 of Refs. 46 and 48. Stated in other words, classical mechanics agrees with quantum mechanics in finding a predominantly regular dynamics, provided that the classical system is investigated for time intervals not longer than, roughly, the density of states. This observation is sufficient to reconcile classical and quantum mechanics, since the correspondence between the two of them is anyway not ex-
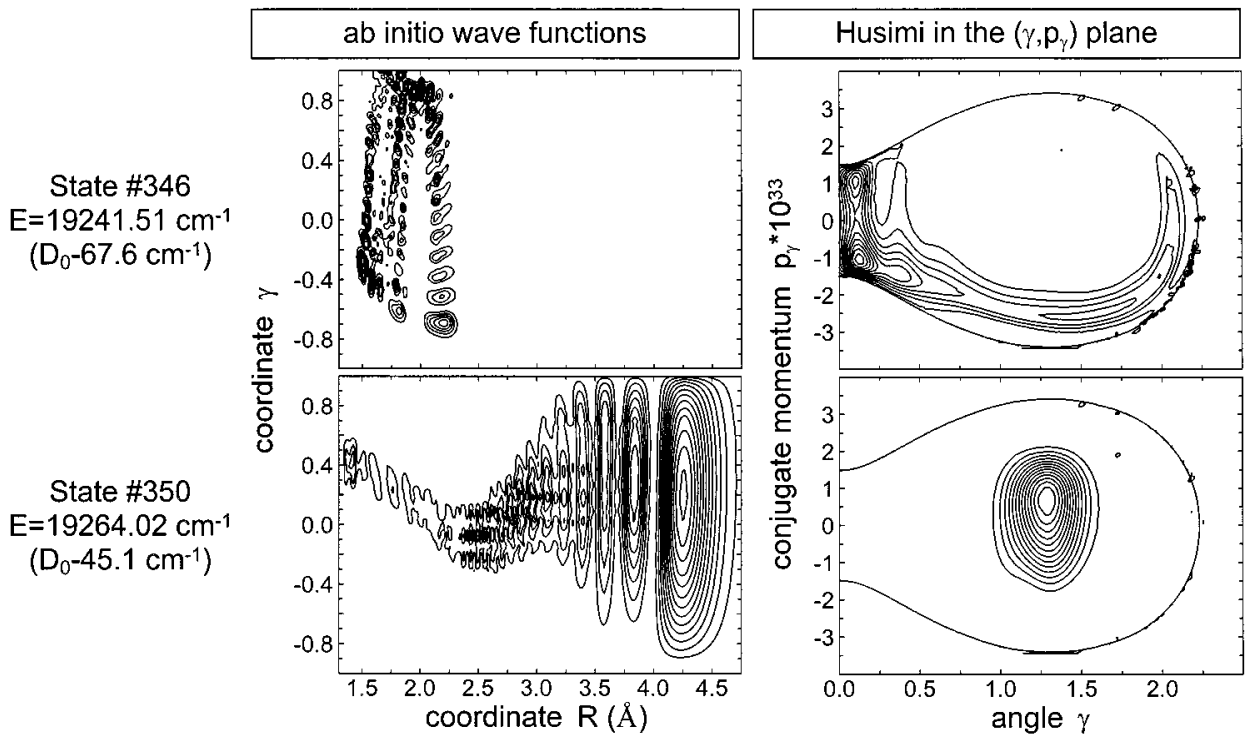

FIG. 7. Plot of quantum wave functions (left) and Husimi distributions (right) for states no. 346 (top) and no. 350 (bottom). State no. 346 is located $67.6 \mathrm{~cm}^{-1}$ below the dissociation threshold and assigned as the state with highest energy in polyad $P=32$. State no. 350 is located $45.1 \mathrm{~cm}^{-1}$ below the dissociation threshold and assigned as the state with lowest energy in polyad $P=45$. Remember that the last bound state is no. 355 . Husimi distributions are plotted as in Fig. 6. 
pected to extend beyond the time corresponding to the density of states.

Things are however very different for $2 D \mathrm{HOCl}$ at $18500 \mathrm{~cm}^{-1}$. In this energy region, the density of states is about 0.052 states $/ \mathrm{cm}^{-1}$, which corresponds to a time of $1700 \mathrm{fs}$, that is, about 50 periods of the unstable bending $\mathrm{PO}(T=34 \mathrm{fs}), 30$ periods of the stable $[R] \mathrm{PO}(T=61 \mathrm{fs})$, or 10 periods of the stable $[D D]$ dissociation $\operatorname{PO}(T=195 \mathrm{fs})$. The point is, that examination of sequential SOSs shows that trajectories remaining confined on "vague" tori during this large amount of time are clearly not the rule. In order to get a deeper insight into the remaining structure of the classical phase space, we additionally performed linear stability analyses along randomly chosen trajectories. ${ }^{50-52}$ The result is that regular and chaotic dynamics usually alternate at roughly equivalent time intervals of about one vibrational period (50-100 fs). This is probably due to the fact that the dynamics of $\mathrm{HOCl}$ is investigated in a comparatively higher energy region compared to the work dealing with the Henon-Heiles surface, so that larger portions of vague tori have been destroyed by chaos.

Conclusions regarding these comparisons between the classical and quantum vibrational dynamics of $2 \mathrm{D} \mathrm{HOCl}$ just below the dissociation threshold can therefore be expressed in three ways. (i) First, it can be considered that classical mechanics is a valid approximation of quantum mechanics only over the time interval where classical mechanics looks regular (about one vibrational period), that is, one to two orders-of-magnitude shorter than the (commonly assumed) density of states. (ii) On the other hand, it can be remarked that the principal features of the quantum states are governed by the very small islands of regularity surrounding stable POs and not by the much larger chaotic regions surrounding these islands. Points (i) and (ii) are fully consistent with Eckardt et al.'s remark that 'it suffices to stay in the vicinity of a PO one period for quantum effects to become important." 49 (iii) At last, it should be noticed that the case of $2 \mathrm{D} \mathrm{HOCl}$ is exactly parallel to the case of $\mathrm{HCN}$, for which some controversy arose as it appeared that the classical dynamics is much more chaotic than the quantum dynamics. ${ }^{53-58}$ Most interestingly, it has been shown very recently that the $\mathrm{HCN} / \mathrm{CNH}$ system, just like $\mathrm{HOCl}$, remains very close (an average $10 \mathrm{~cm}^{-1}$ deviation) to an integrable (i.e., nonchaotic) system up to the isomerization threshold. ${ }^{59}$ In both cases, chaos therefore appears as a small fluctuation (a few $\mathrm{cm}^{-1}$ ) around a basically integrable system, even in the case where chaos is fully developed and occupies all the available phase space. The studies dealing with $\mathrm{HCN}$ and $\mathrm{HOCl}$ suggest that quantum dynamics is largely unsensitive to the small chaotic fluctuation.

\section{B. Intramolecular vibrational energy redistribution (IVR)}

In addition to displaying overall regularity, the Husimi distributions in Fig. 6 also show a very clear separation of the phase space into two domains, which are just the "pit", and the "pulp" of the avocado. By comparison with the quantum wave functions in Fig. 2 and the classical surfaces of section in Fig. 5, these two domains are clearly assigned

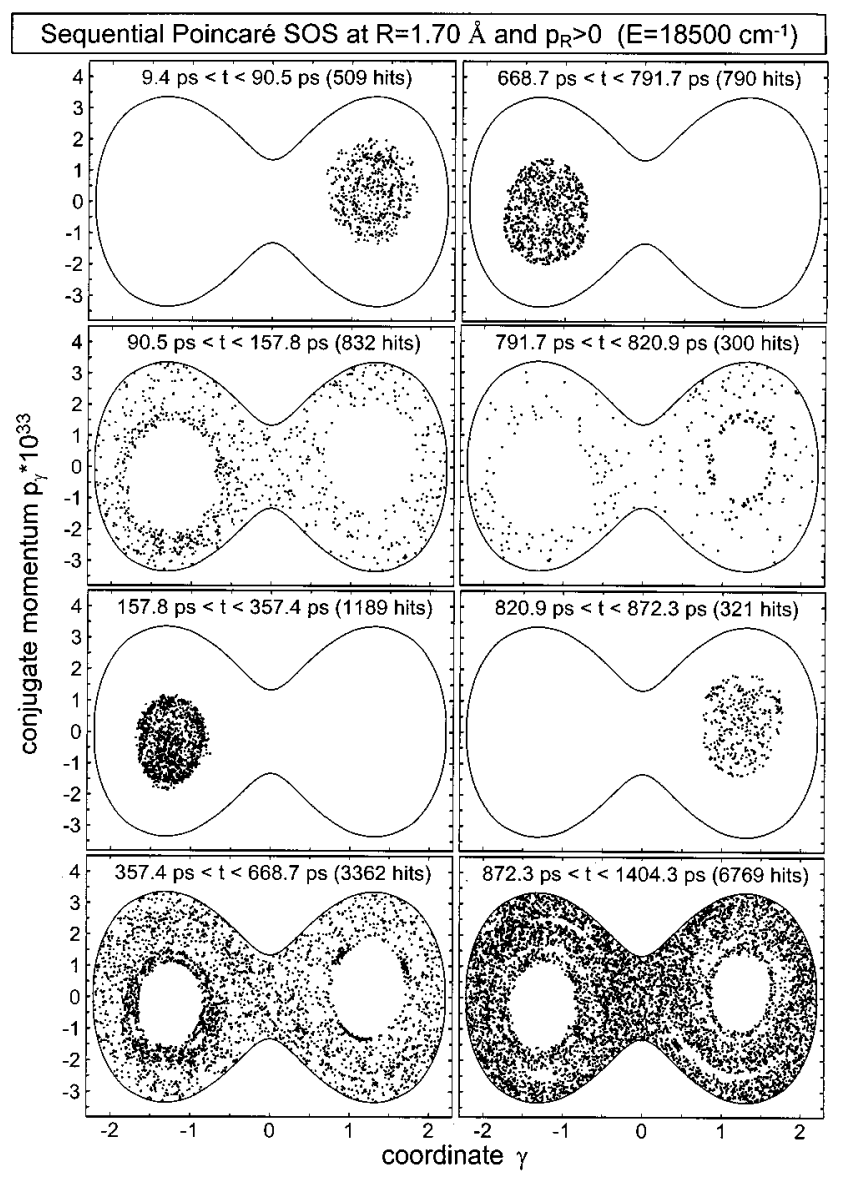

FIG. 8. Sequential Poincare surfaces of section in the $\left(\gamma, p_{\gamma}\right)$ plane at $18500 \mathrm{~cm}^{-1}$ above the quantum mechanical ground state. The eight diagrams show the intersections with the plane $R=1.70 \AA$ of a single trajectory integrated for more than $1.4 \mathrm{~ns}$. The total integration time is however split into eight intervals, in order for the slow IVR process discussed in Sect. IV $\mathrm{B}$ to appear clearly. For the sake of clarity, the two equivalent wells connected through the linear $\mathrm{HOCl}$ configuration $(\gamma=0)$ have been shown.

to "dissociation" and "nondissociation" motions, respectively, where dissociation motion again means a motion for which all the energy flows back and forth along the dissociation pathway. The separation of the quantum phase space into two domains persists up to the dissociation threshold, as can be checked in Fig. 7. This figure shows the wave functions (left column) and the Husimi distributions (right column) for states no. 346 and 350, which are assigned as the highest state of polyad $P=32$ and the lowest state of polyad $P=45$, respectively. These states are located at 68 and 45 $\mathrm{cm}^{-1}$ below the dissociation threshold (remember that the last bound state is no. 355). This figure shows that, even just a few tens of $\mathrm{cm}^{-1}$ below the threshold, energy does not flow easily from the dissociation pathway to perpendicular directions and conversely.

This slow intramolecular vibrational energy redistribution (IVR) also appears very clearly when plotting sequential SOSs as in Fig. 8. This figure shows the intersections with the plane $R=1.70 \AA$ of a single trajectory integrated for more than $1.4 \mathrm{~ns}$ at an energy of $18500 \mathrm{~cm}^{-1}$ above the quantum ground state. Instead of plotting all of the 14000 points on the same diagram, as in the left column of Fig. 5, 
the total integration time is however split into eight intervals, in order for the slow IVR process to become obvious. This figure shows that the trajectory spends a pretty large amount of time - in the range 30 to $500 \mathrm{ps-in} \mathrm{each} \mathrm{region} \mathrm{of} \mathrm{the}$ phase space (pit or pulp) before jumping into the other region. Such an IVR time constant of about 100 ps is three orders-of-magnitude larger than the average vibrational period (about $100 \mathrm{fs}$ ) and two orders-of-magnitude larger than the density of states (about 1.7 ps). Keeping with Reinhardt's idea of only partially destroyed tori, observation of such a long IVR time constant implies that, whereas most of the tori have already been substantially destroyed by chaos at 18500 $\mathrm{cm}^{-1}$ (cf. Sec. IV A), some of those located at the frontier between the two regions are nevertheless still almost intact. Linear stability analyses of trajectories launched close to the frontier were able to confirm this viewpoint. Moreover, the explanation in terms of partially broken tori suggests that the average time for crossing the barrier should decrease rapidly with energy, since the "porousness" of the tori increases with energy. Plotting sequential SOSs similar to Fig. 8 at an energy E equal to $19200 \mathrm{~cm}^{-1}$, that is, $100 \mathrm{~cm}^{-1}$ below the dissociation threshold, proves that this is indeed the case: The increase of $700 \mathrm{~cm}^{-1}$ in vibrational energy results in a decrease of the time constant from the $30-500$ ps range to the $1-13$ ps range.

Keeping in mind that the dissociation time constant of $\mathrm{HOCl}$, when all the energy is deposited in the $\mathrm{OH}$ stretch degree of freedom (not taken into account in the present 2D study), is in the range $10-100 \mathrm{~ns},{ }^{15-20}$ one sees that there exist at least two bottlenecks, which prevent energy from flowing freely between the three degrees of freedom and are responsible for the wide distribution of resonance widths found in the quantal calculations: $:^{8,25,28}$ the (by far) narrowest one separates the $\mathrm{OH}$ stretch from the other degrees of freedom, while the second bottleneck found in the present work separates the $\mathrm{OCl}$ stretch (i.e., the dissociation motion) from the bending motion. In order to have a complete picture of the IVR scheme in $\mathrm{HOCl}$, there just remains to understand whether energy escaping the $\mathrm{OH}$ stretch degree of freedom preferentially flows into the $\mathrm{OCl}$ stretch or the bending ones. It is to be noted that similar bottlenecks have also been detected in OCS (Ref. 60) and $\mathrm{HeI}_{2}$ (Ref. 61), where distinction is made between 'intermolecular', bottlenecks (i.e., leading to dissociation) and 'intramolecular', bottlenecks (i.e., between nondissociation modes). The reader is referred to these works for more detailed information.

\section{ACKNOWLEDGMENT}

J.M.B. thanks the National Science Foundation (CHE9873571) for support.

\footnotetext{
${ }^{1}$ R. Prosmiti, S. C. Farantos, R. Guantes, F. Borondo, and R. M. Benito, J. Chem. Phys. 104, 2921 (1996).

${ }^{2}$ F. Borondo, A. A. Zembekov, and R. M. Benito, J. Chem. Phys. 105, 5068 (1996).

${ }^{3}$ C. Beck, R. Schinke, and J. Koput, J. Chem. Phys. 112, 8446 (2000).

${ }^{4}$ C. Beck, H. M. Keller, S. Y. Grebenshchikov, R. Schinke, S. C. Farantos, K. Yamashita, and K. Morokuma, J. Chem. Phys. 107, 9818 (1997).

${ }^{5}$ H. Ishikawa, R. W. Field, S. C. Farantos, M. Joyeux, J. Koput, C. Beck, and R. Schinke, Annu. Rev. Phys. Chem. 50, 443 (1999).
}

${ }^{6}$ M. Joyeux, D. Sugny, V. Tyng, M. Kellman, H. Ishikawa, R. W. Field, C. Beck, and R. Schinke, J. Chem. Phys. 112, 4162 (2000).

${ }^{7}$ R. Jost, M. Joyeux, S. Skokov, and J. Bowman, J. Chem. Phys. 111, 6807 (1999).

${ }^{8}$ J. Weiß, J. Hauschildt, S. Y. Grebenshchikov, R. Düren, R. Schinke, J. Koput, S. Stamatiadis, and S. C. Farantos, J. Chem. Phys. 112, 77 (2000).

${ }^{9}$ E. L. Sibert and A. B. McCoy, J. Chem. Phys. 105, 469 (1996).

${ }^{10}$ M. P. Jacobson, C. Jung, H. S. Taylor, and R. W. Field, J. Chem. Phys. 111, 600 (1999).

${ }^{11}$ J. Bredenbeck, C. Beck, R. Schinke, J. Koput, S. Stamatiadis, S. C. Farantos, and M. Joyeux, J. Chem. Phys. 112, 8855 (2000).

${ }^{12}$ R. Prosmiti, S. C. Farantos, and H. Guo, Chem. Phys. Lett. 311, 241 (1999).

${ }^{13}$ B. Abel, H. H. Hamann, A. A. Kachanov, and J. Troe, J. Chem. Phys. 104, 3189 (1996).

${ }^{14}$ B. Abel, A. Charvat, S. F. Deppe, and H. H. Hamman, Ber. Bunsenges. Phys. Chem. 101, 329 (1997).

${ }^{15}$ R. J. Barnes, G. Dutton, and A. Sinha, J. Phys. Chem. 101, 8374 (1997).

${ }^{16}$ R. J. Barnes and A. Sinha, J. Chem. Phys. 107, 3730 (1997).

${ }^{17}$ A. Callegari, J. Rebstein, J. S. Muenter, R. Jost, and T. R. Rizzo, J. Chem. Phys. 111, 123 (1999); ibid. 112, 2569 (2000).

${ }^{18}$ A. Callegari, J. Rebstein, R. Jost, and T. R. Rizzo, J. Chem. Phys. 111, 7359 (1999)

${ }^{19}$ M. R. Wedlock, R. Jost, and T. R. Rizzo, J. Chem. Phys. 107, 10344 (1997).

${ }^{20}$ J. S. Muenter, J. Rebstein, A. Callegari, and T. R. Rizzo, J. Chem. Phys. 111, 3488 (1999).

${ }^{21}$ S. Skokov, K. A. Peterson, and J. M. Bowman, J. Chem. Phys. 109, 2662 (1998).

${ }^{22}$ S. Skokov, J. Qi, J. M. Bowman, C. Y. Yang, S. K. Gray, K. A. Peterson, and V. A. Mandelshtam, J. Chem. Phys. 109, 10273 (1998).

${ }^{23}$ S. Skokov, J. M. Bowman, and V. A. Mandelshtam, Phys. Chem. Chem. Phys. 1, 1279 (1999).

${ }^{24}$ S. Skokov and J. M. Bowman, J. Chem. Phys. 110, 9789 (1999).

${ }^{25}$ S. Skokov and J. M. Bowman, J. Chem. Phys. 111, 4933 (1999).

${ }^{26}$ K. A. Peterson, S. Skokov, and J. M. Bowman, J. Chem. Phys. 111, 7446 (1999).

${ }^{27}$ S. Skokov, K. A. Peterson, and J. M. Bowman, Chem. Phys. Lett. 312, 494 (1999).

${ }^{28}$ J. Hauschildt, J. Weiß, S. Yu. Grebenshchikov, R. Düren, R. Schinke, and J. Koput, Chem. Phys. Lett. 300, 569 (1999).

${ }^{29}$ J. Hauschildt, J. Weiß, and R. Schinke, Z. Phys. Chem. (Munich) 214, 609 (2000).

${ }^{30}$ S. C. Farantos, Comput. Phys. Commun. 108, 240 (1998).

${ }^{31}$ M. Founargiotakis, S. C. Farantos, G. Contopoulos, and C. Polymilis, J. Chem. Phys. 91, 1389 (1989).

${ }^{32}$ M. Joyeux, J. Phys. A 29, 5963 (1996).

${ }^{33}$ M. Joyeux and L. Michaille, ACH-Models Chem. 134, 573 (1997).

${ }^{34}$ A. Einstein, Verh. Dtsch. Phys. Ges. 19, 82 (1917).

${ }^{35}$ J. B. Keller, Ann. Phys. (N.Y.) 4, 180 (1958).

${ }^{36}$ V. P. Maslov and M. V. Fedoriuk, Semiclassical Approximations in Quantum Mechanics (Deidel, Dordrecht, 1981).

${ }^{37}$ K. Husimi, Proc. Phys. Math. Soc. Jpn. 22, 264 (1940).

${ }^{38}$ K. Takahashi, J. Phys. Soc. Jpn. 55, 762 (1986).

${ }^{39}$ Y. Weissman and J. Jortner, J. Chem. Phys. 77, 1486 (1982).

${ }^{40}$ K. Takahashi and N. Saito, Phys. Rev. Lett. 55, 645 (1985).

${ }^{41}$ M. J. Davis, J. Chem. Phys. 107, 106 (1997).

${ }^{42}$ M. J. Davis, J. Chem. Phys. 107, 4507 (1997).

${ }^{43}$ C. Jaffé and W. P. Reinhardt, J. Chem. Phys. 71, 1862 (1979).

${ }^{44}$ C. Jaffé and W. P. Reinhardt, J. Chem. Phys. 77, 5191 (1982).

${ }^{45}$ R. B. Shirts and W. P. Reinhardt, J. Chem. Phys. 77, 5204 (1982).

${ }^{46}$ W. P. Reinhardt, J. Phys. Chem. 86, 2158 (1982).

${ }^{47}$ W. P. Reinhardt and D. Farrelly, J. Phys. (France) 43, C2-29 (1982).

${ }^{48}$ K. Sohlberg and R. B. Shirts, J. Chem. Phys. 101, 7763 (1994).

${ }^{49}$ B. Eckhardt, G. Hose, and E. Pollak, Phys. Rev. A 39, 3776 (1989).

${ }^{50}$ P. Brumer and J. W. Duff, J. Chem. Phys. 65, 3566 (1976).

${ }^{51}$ C. Cerjan and W. P. Reinhardt, J. Chem. Phys. 71, 1819 (1979).

${ }^{52}$ R. Kosloff and S. A. Rice, J. Chem. Phys. 74, 1947 (1981).

${ }^{53}$ K. K. Lehmann, G. J. Scherer, and W. Klemperer, J. Chem. Phys. 76, 6441 (1982).

${ }^{54}$ K. K. Lehmann, G. J. Scherer, and W. Klemperer, J. Chem. Phys. 77, 2853 (1982). 
${ }^{55}$ D. Farrelly and W. P. Reinhardt, J. Chem. Phys. 78, 606 (1983).

${ }^{56}$ K. K. Lehmann, G. J. Scherer, and W. Klemperer, J. Chem. Phys. 78, 608 (1983).

${ }^{57}$ M. Founargiotakis, S. C. Farantos, and J. Tennyson, J. Chem. Phys. 88, 1598 (1988).
${ }^{58}$ S. C. Farantos, J. M. Gomez Llorente, O. Hahn, and H. S. Taylor, J. Chem. Phys. 93, 76 (1990).

${ }^{59}$ D. Sugny, M. Joyeux, and E. L. Sibert III, J. Chem. Phys. (in press).

${ }^{60}$ M. J. Davis, J. Chem. Phys. 83, 1016 (1985).

${ }^{61}$ M. J. Davis and S. K. Gray, J. Chem. Phys. 84, 5389 (1986). 